Renato Ludmer Guedes Alcoforado ${ }^{1}$ Adriana Conrado de Almeida ${ }^{1}$ Gabriela Granja Porto ${ }^{1}$ Jackellyne Carneiro da Silva ${ }^{1}$ Antonio Azoubel Antunes ${ }^{1}$

\title{
A JUDICIALIZAÇÃO DE CIRURGIA BUCOMAXILOFACIAL E O SISTEMA DE ASSISTÊNCIA À SAÚDE DOS SERVIDORES PÚBLICOS DE PERNAMBUCO
}

The judicialization of oral and maxillofacial surgery and the Health Assistance System of Public Servants in Pernambuco

${ }^{1}$ Universidade de Pernambuco. Recife/PE, Brasil

Correspondência: Renato Ludmer Guedes Alcoforado. E-mail: renato.ludmer@upe.br

Recebido: 02/09/2018. Revisado: 12/06/2019. Nova revisão: 09/09/2019. Aprovado: 16/10/2019. 


\section{RESUMO}

A Constituição Federal de 1988 foi pioneira ao dispor sobre o direito à saúde como direito fundamental e regular o sistema suplementar de saúde. Vários estados brasileiros criaram planos voltados exclusivamente para seus servidores, e Pernambuco foi o pioneiro a concebê-los, através do Sistema de Assistência à Saúde dos Servidores do Estado de Pernambuco. Os avanços tecnológicos, o aumento da expectativa de vida (e a consequente perda dentária precoce) e os traumas provocaram aumento do uso de órteses, próteses e materiais especiais em procedimentos invasivos - proporcionando, dentre outros benefícios, a reparação estética. Porém, há entraves ao uso desses materiais, entre eles seu alto custo e a ausência de cobertura dos planos de saúde para procedimentos estéticos. Foram analisados os processos judiciais envolvendo órteses, próteses e materiais especiais em cirurgia bucomaxilofacial contra o Sistema de Assistência à Saúde dos Servidores do Estado de Pernambuco, no período de janeiro de 2009 a dezembro de 2017. O propósito foi defender que a indicação do material a ser utilizado pelo cirurgião-dentista não pode ser considerada critério absoluto e que o Poder Judiciário não deve acatar tal opinião sem questioná-la. Verificou-se a necessidade de franquear maior estrutura aos magistrados, com a criação de núcleos de assistência técnica em saúde e/ou investimentos nos já existentes, a fim de subsidiar os julgadores e assegurar uma maior eficiência na solução das demandas. A metodologia aplicada foi a do estudo transversal, baseado na análise de dados quantitativos e qualitativos extraídos dos processos. Como variáveis, traçaram-se o perfil dos autores das ações e os parâmetros adotados nas decisões judiciais.

\section{Palavras-Chave}

Assistência à Saúde; Judicialização da Saúde; Saúde Suplementar.

\section{ABSTRACT}

The Brazilian Federal Constitution of 1988 was a pioneer in providing the right to health as a fundamental right and regulating the supplementary health system. Several states have created plans aimed exclusively at their servants, and Pernambuco was the pioneer to conceive them, through the Health Assistance System of the Servants of the State of Pernambuco. The technological advances, the increase of life expectancy (and the consequent early tooth loss) and the traumas have caused an increase in the use of orthoses, prostheses and special materials in invasive procedures - providing, among other benefits, esthetic repair. However, there are obstacles to the use of these materials, including their high cost and the lack of private health insurance coverage for esthetic procedures. The judicial proceedings involving orthoses, prostheses and special materials in oral and maxillofacial surgery against the Health Assistance System of the Servants of the State of Pernambuco were analyzed, from January 2009 to December 2017. The purpose was to defend that the indication of the material to be used by the dental surgeon cannot be considered as an absolute criterion and that the Judiciary should not accept such an opinion without questioning it. It was verified the need to open a larger structure to the magistrates, with the creation of centers for technical assistance in health and/or investments in the existing one, in order to subsidize the judges and ensure a greater efficiency in the solution of the demands. The applied methodology was the cross-sectional study, based on the analysis of quantitative and qualitative data extracted from the processes. As variables, the profile of the authors of the actions and the parameters adopted in the judicial decisions were traced.

\section{Keywords}

Health Assistance; Judicialization of Health; Private Health Insurance. 


\section{Introdução}

A Carta Constitucional de $1988(\mathrm{CF} / 88)^{1}$ elencou a saúde como direito fundamental e dever do Estado, fornecida por meio de um sistema unificado com o propósito de garantir à população uma assistência integral e totalmente gratuita. Essa integralidade deve ser analisada em vários níveis de discussão e prática na área de saúde, como fundamento de um novo paradigma para atender às demandas e necessidades de toda a coletividade ${ }^{2}$.

Tendo em vista a impossibilidade financeira de o Sistema Único de Saúde (SUS) atender toda a população ${ }^{3}$, tem-se verificado a busca por assistência médica suplementar, que, no caso dos servidores públicos e seus dependentes, é oferecida por planos específicos nos quais há uma contrapartida do ente público e do servidor a ele vinculado. A majoração desses planos deve-se à crise econômica e política por qual passa nosso país, razão pela qual muitos servidores migraram para planos copatrocinados pelos entes públicos aos quais estão vinculados ou tiveram que recorrer ao sistema público de saúde.

Os anos de 2017 e 2018 foram marcados por seguidas reviravoltas nos rumos da economia brasileira: tendo começado com expectativas positivas de recuperação das atividades econômicas, muito retraídas desde a grande recessão de 2014-2016, problemas internos abateram a retomada e o desempenho anual ficou muito aquém do que se esperava ${ }^{4}$.

Nesse diapasão, os planos e seguros de saúde tiveram uma perda significativa de usuários. Segundo a Agência Nacional de Saúde Suplementar (ANS) ${ }^{5}$, o total de beneficiários passou de 50.444.761 em dezembro de 2014 para $47.133 .325 \mathrm{em}$ abril de 2019. Assim, nesse intervalo de cinco anos, mais de 3 milhões de pessoas (3.311.436) deixaram o sistema privado de saúde, com repercussão direta no orçamento e na gestão do já precário SUS.

De outro giro, Pernambuco foi o primeiro estado a criar um instrumento próprio de assistência médica para os servidores públicos estaduais. O Sistema de

\footnotetext{
${ }^{1}$ BRASIL. Constituição da República Federativa do Brasil de 1988. Disponível em: http://www.planalto.gov. br/ccivil_03/constituicao/constituicaocompilado.htm. Acesso em: 16 fev. 2021.

${ }^{2} \mathrm{UCHOA}$, Severina Alice da Costa; COSTA, Isabelle Katherinne Fernandes; DANTAS, Rodrigo Assis Neves. Os sentidos da integralidade da assistência à saúde: uma revisão da literatura. InterSciencePlace, v. 1, n. 12, p. 78-91, 2015. Disponível em: http://www.interscienceplace.org/isp/index.php/isp/article/ download/121/120.

${ }^{3}$ LIMA, Júlio César França. Neoliberalismo e formação profissional em saúde. Revista Trabalho Necessário, v. 4, n. 4, jun. 2018. Disponível em: https://periodicos.uff.br/trabalhonecessario/article/view/4613/4249. https://doi.org/10.22409/tn.5i5.p4613.

${ }^{4}$ FUNDAÇÃO GETÚLIO VARGAS - FGV. 2018. FGV e Instituto Brasileiro de Econômica. Maio 2019. Disponível em https://portalibre.fgv.br/data/files/30/F2/67/E0/EE4EA61078ADFDA68904CBA8/ BoletimMacrolbre_1905.pdf. Acesso em: 07 jun. 2019.

${ }^{5}$ ANS disponibiliza números de abril do setor de planos de saúde. Notícias ANS, 05 jun. 2019. Disponível em: http://www.ans.gov.br/aans/noticias-ans/numeros-do-setor/5009-ans-disponibiliza-numeros-de-abr il-do-setor-de-planos-de-saude. Acesso em: 07 jun. 2019.
} 
Assistência à Saúde dos Servidores do Estado de Pernambuco (SASSEPE) foi criado pela Lei Complementar n. 30, de 2 de janeiro de 2001, com o propósito de realizar ações de medicina preventiva e curativa, ambulatorial e hospitalar, por meio de entidades, profissionais e hospitais credenciados e por rede própria ${ }^{6}$.

O SASSEPE consiste em um sistema de adesão voluntária e conta com a participação dos beneficiários no custeio, além de contribuição fixa do Poder Executivo estadual. Diferencia-se dos seguros saúde existentes no mercado por ser exclusivo dos servidores estaduais e seus dependentes, não ter finalidade lucrativa e não estar subordinado ao poder regulador da $\mathrm{ANS}^{7}$.

Observa-se, nos últimos anos, um aumento das demandas judiciais envolvendo o fornecimento de órteses, próteses e materiais especiais (OPMEs) ${ }^{8}$, geralmente usados em cirurgias ortognáticas e reconstrutivas dos maxilares, as quais podem ser consideradas procedimentos estético-funcionais para o tratamento das deformidades dentais. Objetivam a correção de deficiências no paciente e trazem, na maioria dos casos, grande satisfação pessoal ${ }^{9}$.

Em muitos casos, o Poder Judiciário tem determinado liminarmente a entrega de OPMEs cuja cobertura não tem sequer previsão contratual, baseando-se apenas no pedido do profissional de saúde assistente da parte autora ${ }^{10}$, sob o argumento de que cabe a ele determinar o material adequado para o tratamento do paciente.

O Conselho Federal de Odontologia (CFO) elaborou a Resolução n. $115 / 2012^{11}$, que proibiu o cirurgião-dentista de exigir fornecedor ou marca exclusiva, cabendo ao profissional determinar apenas as características principais dos OPMEs necessários à execução do procedimento almejado. Deve o profissional, nesse caso, oferecer às instituições públicas ou privadas pelo menos três marcas de produtos,

${ }^{6}$ INSTITUTO DE RECURSOS HUMANOS DE PERNAMBUCO - IRH. Sistema de Assistência à Saúde dos Servidores Públicos do Estado de Pernambuco. Disponível em http://www.irh.pe.gov.br/web/irh/sassepe. Acesso em: 10 jun. 2017.

${ }^{7}$ BRASIL. Lei n. 9.961, de 28 de janeiro de 2000. Cria a Agência Nacional de Saúde Suplementar - ANS e dá outras providências. Disponível em: http://www.planalto.gov.br/ccivil_03/leis/I9961.htm\#: :text=LEI\%20 No\%209.961\%20DE\%2028\%20DE\%2OJANEIRO\%20DE\%202000.\&text=Cria\%20a\%20Ag\%C3\%AAncia\%20 Nacional\%20de,ANS\%20e\%20d\%C3\%A1\%20outras\%20provid\%C3\%AAncias. Acesso em: 16 fev. 2021.

${ }^{8}$ ALENCAR, Anna Carolyne Ferreira. Aquisição e utilização das órteses, próteses e materiais especiais-OPME e os facilitadores do superfaturamento no sistema de saúde. 2016. Monografia (Bacharelado em Gestão em Saúde Coletiva) - Universidade de Brasília, Brasília-DF, 2016.

${ }^{9}$ LAUREANO, José Rodrigues et al. Alterações em discrepância ântero-posterior na cirurgia ortognática. Rev. Cir. Traumatol. Buco-Maxilo-Fac. Camaragibe, v. 5, n. 1, p. 45-52, jan./mar. 2005. Disponível em: https:// www.revistacirurgiabmf.com/2005/v5n1/pdf\%20v5n1/artigo\%2007.pdf.

${ }^{10}$ MARTINS, Paulo Roberto do Nascimento; DAHINTEN, Bernardo Franke; DAHINTEN, Augusto Franke. 0 problema envolvendo as OPMES e os planos de saúde: contornos e análise da problemática. Revista de Direito Sanitário, São Paulo, v. 17, n. 1, p. 145-166, 2016. Disponível em: https://www.revistas.usp.br/ rdisan/article/view/117052/114650. https://doi.org/10.11606/issn.2316-9044.v17i1p145-166.

${ }^{11}$ CONSELHO FEDERAL DE ODONTOLOGIA. Resolução n. 115, de 03 de abril de 2012. Disponível em: <http:// http://www.crosp.org.br/uploads/paginas/05c3dd25d7da8f204e6bfed435990cfb.pdf. Acesso em: 01.082018. 
de fabricantes diferentes e regularizadas junto à Agência Nacional de Vigilância Sanitária (Anvisa). O cirurgião também deve seguir os parâmetros e recomendações do Colégio Brasileiro de Cirurgia e Traumatologia Bucomaxilofacial, que servem de referência para a utilização de abordagens, procedimentos, órteses, próteses e materiais especiais no exercício da profissão ${ }^{12}$.

A imprensa tem noticiado práticas ilícitas de alguns profissionais de saúde envolvendo a indicação e utilização de marca comercial de órteses, próteses e materiais especiais ${ }^{13}$. Tal comportamento resulta da crescente mercantilização da saúde, por sua vez resultante do assédio financeiro de alguns fabricantes, os quais oferecem vantagens pecuniárias em troca da utilização de marcas específicas, independentemente da efetiva indicação técnica e da necessidade do usuário.

Diante desse cenário, constata-se o conflito de interesses entre o usuário/ médico assistente e o sistema de saúde - notadamente quando há uma indicação, por parte do profissional, de determinada marca de $\mathrm{OPME}^{14}$-, trazendo a discussão sobre a judicialização em relação ao fornecimento de tais dispositivos.

Segundo o Conselho Nacional de Justiça $(\mathrm{CNJ})$, o número de ações judiciais relativas à saúde aumentou $130 \%$ entre 2008 e $2017^{15}$. Esse crescimento exacerbado gera reflexos constitucionais e orçamentários sob a perspectiva da gestão ${ }^{16}$, na medida em que gera um aumento significativo das despesas públicas e na contabilidade dos planos e seguros de saúde. A complexidade do tema sinaliza para um cenário desigual do acesso da população a bens e serviços de saúde, com consequências relevantes na relação com os direitos fundamentais ${ }^{17}$.

A pesquisa acerca das ações judiciais tem se mostrado um importante campo de investigação na área da saúde. O estudo de seus aspectos políticos, sociais, éticos, jurídicos e sanitários pode trazer elementos para o aprimoramento das políticas de

${ }^{12}$ COLÉGIO BRASILEIRO DE CIRURGIA E TRAUMATOLOGIA BUCO-MAXILO-FACIAL. Parâmetros e recomendações para procedimentos buco-maxilo-faciais do Colégio Brasileiro de Cirurgia e Traumatologia Buco-Maxilo-Facial. Ênfase em OPME. Versão 2017/2018. Edição Compacta. Disponível em: https://www.bucomaxilo.org.br/ upfiles/downloads/diretrizes-e-recomendacoes-para-procedimentos-bmf.pdf. Acesso em: 16 fev. 2021.

${ }^{13}$ MÁFIA das próteses coloca vidas em risco com cirurgias desnecessárias. G1, 04 jan. 2015. Disponível em: http://g1.globo.com/fantastico/noticia/2015/01/mafia-das-proteses-coloca-vidas-em-risco-com-cirurgias -desnecessarias.html. Acesso em: 10 jun. 2017.

${ }^{14}$ Id. Ibid.

${ }^{15}$ CONSELHO NACIONAL DE JUSTIÇA - CNJ. Relatório analítico propositivo sobre a judicialização da saúde no Brasil. Brasília-DF: CNJ, maio, 2019. Disponível em: http://www.cnj.jus.br/files/conteudo/ arquivo/2019/03/f74c66d46cfea933bf22005ca50ec915.pdf. Acesso em: 27 maio 2019.

${ }^{16}$ SOUZA, Júlio César de; GOMES, Magno Federici. A judicialização na saúde e a fronteira entre o individual e o coletivo: consideraçõessobre oacessoaoSistemaÚnico deSaúdesustentável. Revista Direitos Fundamentais \& Democracia, v. 24, n. 1, p. 216-242, 2019. Disponível em: https://revistaeletronicardfd.unibrasil.com.br/ index.php/rdfd/article/view/1227/579. https://doi.org/10.25192/issn.1982-0496.rdfd.v24i11227.

${ }^{17}$ RIBEIRO, Krishina Day; VIDAL, Josep Pont. Uma análise da produção acadêmica sobre a evolução do fenômeno da judicialização da saúde no Brasil. Cadernos Ibero-Americanos de Direito Sanitário, v.7, n.2, p.239-261, abr./jun. 2018. Disponivel em: https://www.cadernos.prodisa.fiocruz.br/index.php/cadernos/ article/view/493/548. http://dx.doi.org/10.17566/ciads.v7i2.493. 
saúde em curso e, especificamente no caso da assistência médica suplementar, para a melhoria de sua regulação ${ }^{18}$. Dentre as várias dimensões ${ }^{19}$ de análise da judicialização da saúde, destacam-se aquelas que estudam a atuação do Poder Judiciário, a conformidade com a legislação, as características dos demandantes e os fundamentos das decisões dos magistrados. Por meio do estudo das ações judiciais, é possível mapear os problemas causados pela interferência do Judiciário no sistema de saúde e analisar as eventuais falhas não só do mesmo, mas da própria prestação jurisdicional.

Tendo como norte a crescente discussão sobre a judicialização da saúde e a ausência de publicações sobre o tema, buscou-se analisar a percepção do Poder Judiciário estadual no julgamento dos processos contra o SASSEPE em relação ao acesso a cirurgia bucomaxilofacial com uso de OPMEs. Avaliou-se o interesse dos autores das ações; registraram-se os parâmetros adotados nas decisões judiciais e suas dificuldades com os meios que poderão ser relevantes para minimizar os eventuais óbices enfrentados e propor soluções.

\section{Metodologia}

Trata-se de estudo descritivo, transversal e retrospectivo, com abordagem qualitativa e quantitativa, desenvolvido com os dados do SASSEPE. Foram selecionadas as decisões proferidas liminarmente pelos magistrados das varas da Fazenda Pública da comarca de Recife (PE) e Caruaru (PE), ambas integrantes da justiça comum estadual, no período de janeiro de 2009 a dezembro de 2017. A população foi constituída por beneficiários e seus dependentes com demanda judicial para o fornecimento de OPMEs em procedimentos cirúrgicos na área bucomaxilofacial. Os dados coletados foram obtidos junto à Diretoria Jurídica do SASSEPE. Posteriormente, no sítio eletrônico do Tribunal de Justiça de Pernambuco (ícone "CONSULTAS - processos $1^{\circ}$ grau"), foram acessados os processos judiciais eletrônicos $(\mathrm{PJe})$ por meio do certificado digital concedido pela Ordem dos Advogados do Brasil (OAB). Um questionário estruturado foi aplicado a todas as decisões selecionadas, com a finalidade de traçar o perfil dos autores das ações, selecionar os dados relacionados a seus pedidos e os inerentes aos processos. Em seguida, as informações foram digitadas no programa Microsoft Excel 2016. O programa utilizado para obtenção dos cálculos estatísticos foi o IBM SPSS, na versão 23. A pesquisa foi desenvolvida de acordo com a Resolução n. 466/2012 do Conselho Nacional de Saúde (CNS), CAEE n. 79482517.5.000.5192.

\footnotetext{
${ }^{18}$ ABADE, Erik Asley Ferreira. Judicialização da assistência médica suplementar: um estudo de caso em Pernambuco. 2015. Dissertação (Mestrado). Programa de Pós-Graduação em Saúde Coletiva, Universidade Federal da Bahia, ISC-UFBA, Salvador-BA, 2015. Disponível em: http://repositorio.ufba.br/ri/handle/ ri/1791. Acesso em: 08 jun. 2018.

${ }^{19}$ SCHEFFER, Mário. Coberturas assistenciais negadas pelos planos e seguros de saúde em ações julgadas pelo Tribunal de Justiça do Estado de São Paulo. Revista de Direito Sanitário, São Paulo, v. 14, n. 1, p. 122-131, 2013. Disponivel em: https://www.revistas.usp.br/rdisan/article/view/56627/59644. https://doi.org/10.11606/issn.2316-9044.v14i1p122-131.
} 


\section{Resultados}

A Tabela 1 apresenta os resultados relativos ao perfil demográfico dos autores. Destacam-se os seguintes dados: a idade variou de 21 a 80 anos, com média de 52,29 anos, desvio padrão de 17,42 anos e mediana igual a 54,00 anos; dos 17 autores, nenhum tinha menos de 21 anos nem estava na faixa etária de 31 a 40 anos, 17,6\% tinham de 21 a 30 anos, 23,5\% tinham de 41 a 50 anos, 29,4\% tinham de 51 a 60 anos e 29,4\% tinham 61 anos ou mais; a maioria (82,4\%) era do sexo feminino; mais da metade $(58,8 \%)$ residia em Recife e os $41,2 \%$ restantes, no interior de Pernambuco.

Tabela 1. Avaliação do perfil demográfico dos autores

\begin{tabular}{|c|c|}
\hline Variável & Grupo total \\
\hline TOTAL: n (\%) & $17(100,0)$ \\
\hline Idade: Média \pm DP (Mediana) & $52,29 \pm 17,42(54,00)$ \\
\hline Faixa etária (anos) & $\mathrm{n}(\%)$ \\
\hline$<21$ & - \\
\hline 21 a 30 & $3(17,6)$ \\
\hline 31 a 40 & - \\
\hline 41 a 50 & $4(23,5)$ \\
\hline 51 a 60 & $5(29,4)$ \\
\hline 61 ou mais & $5(29,4)$ \\
\hline Sexo & $\mathrm{n}(\%)$ \\
\hline Masculino & $3(17,6)$ \\
\hline Feminino & $14(82,4)$ \\
\hline Procedência & $\mathrm{n}(\%)$ \\
\hline Recife & $10(58,8)$ \\
\hline Interior & $7(41,2)$ \\
\hline Tempo de plano: Média \pm DP (Mediana) & $24,55 \pm 9,70(25,00)$ \\
\hline Tempo de plano (anos) & $\mathrm{n}(\%)$ \\
\hline 0 a 5 & $1(5,9)$ \\
\hline 6 a 25 & $6(35,3)$ \\
\hline 26 a 40 & $4(23,5)$ \\
\hline Não informado & $6(35,3)$ \\
\hline Valor médio contratado mensal: Média \pm DP (Mediana) & $157,16 \pm 89,53(150,00)$ \\
\hline Valor contratado mensal (reais) & $\mathrm{n}(\%)$ \\
\hline Até 50 reais & $1(5,9)$ \\
\hline 51 a 100 & $3(17,6)$ \\
\hline 101 a 150 & $1(5,9)$ \\
\hline 151 a 200 & - \\
\hline Acima de 200 & $4(23,5)$ \\
\hline Não informado & $8(47,1)$ \\
\hline
\end{tabular}


O valor contratado estava ausente em oito $(47,1 \%)$ autores das ações; no restante, variou de $\mathrm{R} \$ 41,00$ a $\mathrm{R} \$ 218,00$, com média de $\mathrm{R} \$ 157,16$, desvio padrão de $\mathrm{R} \$ 89,53$ e mediana igual a $\mathrm{R} \$ 150,00$.

Dos resultados contidos na Tabela 2, ressalta-se que: dos três tipos de procedimentos solicitados, os dois mais frequentes foram cirurgia ortognática $(47,1 \%)$ e cirurgia reconstrutiva $(41,2 \%)$, e os $11,8 \%$ restantes corresponderam a tratamento de patologias; a hipótese diagnóstica mais frequente foi dentoesquelética, em 52,9\% dos casos, seguida de atrofia óssea dos maxilares $(29,4 \%)$ e de patologia dos maxilares $(17,6 \%)$; apenas um cirurgião $(5,9 \%)$ era credenciado ao SASSEPE; a presença de cobertura contratual foi registrada em $23,5 \%$ das ações; em $41,2 \%$, o procedimento não foi realizado e os demais foram realizados em hospital credenciado; em todos os 17 casos, houvera indicação do fornecedor pelo cirurgião; a maioria $(64,7 \%)$ dos casos teve negativa do requerimento pelo SASSEPE; uma segunda opinião ocorreu em 52,9\% dos casos; o valor do procedimento variou de R\$28.380,00 a R\$ 251.901,60, com média de $\mathrm{R} \$ 144.494,56$, desvio padrão de $\mathrm{R} \$ 76,265,28$ e mediana igual a $\mathrm{R} \$ 129.000,00$; a maioria $(58,8 \%)$ dos procedimentos apresentou custo superior a $\mathrm{R} \$ 70.000,00$, e as faixas de $\mathrm{R} \$ 1.000,00$ a $\mathrm{R} \$ 30.000,00$, de $\mathrm{R} \$ 30.001,00$ a $\mathrm{R} \$ 50.000,00$ e de $\mathrm{R} \$ 50.001,00$ a 70.000,00 tiveram frequências unitárias.

Tabela 2. Avaliação dos dados relacionados ao procedimento

\begin{tabular}{lc}
\hline Variável & Grupo total \\
\hline TOTAL: $n$ (\%) & $17(100,0)$ \\
\hline Procedimento solicitado & $\mathrm{n}(\%)$ \\
\hline Cirurgia ortognática & $8(47,1)$ \\
Cirurgia reconstrutiva & $7(41,2)$ \\
Tratamento de patologias & $2(11,8)$ \\
\hline Hipótese diagnóstica: $\mathrm{n}(\%)$ & $\mathrm{n}(\%)$ \\
\hline Deformidade dentoesquelética & $9(52,9)$ \\
Atrofia óssea dos maxilares & $5(29,4)$ \\
Patologia dos maxilares & $3(17,6)$ \\
\hline Cirurgião credenciado & $\mathrm{n}(\%)$ \\
\hline Sim & $1(5,9)$ \\
Não & $16(94,1)$ \\
\hline Cobertura contratual & $\mathrm{n}(\%)$ \\
\hline Sim & $4(23,5)$ \\
Não & $13(76,5)$ \\
\hline Local do procedimento & $\mathrm{n}(\%)$ \\
\hline Não realizado & $7(41,2)$ \\
Hospital credenciado & $10(58,8)$ \\
\hline
\end{tabular}




\begin{tabular}{lc} 
Continuação & Grupo total \\
\hline Variável & $17(100,0)$ \\
\hline Indicação do fornecedor cirurgião: $\mathrm{n}(\%)$ & $\mathrm{n}(\%)$ \\
\hline Negativa do requerimento administrativo & $11(64,7)$ \\
\hline Sim & $6(35,3)$ \\
Não & $\mathrm{n}(\%)$ \\
\hline Segunda opinião & $9(52,9)$ \\
\hline Sim & $8(47,1)$ \\
Não & $\mathrm{n}(\%)$ \\
\hline Valor do procedimento: Média \pm DP (Mediana) & $1(5,9)$ \\
\hline Valor do procedimento (reais) & $1(5,9)$ \\
\hline 1.000 a 30.000 & $1(5,9)$ \\
30.001 a 50.000 & $10(58,8)$ \\
50.001 a 70.000 & $4(23,5)$ \\
70.001 ou mais & $76.265,28(129.000,00)$ \\
Não informado &
\end{tabular}

Dos resultados relacionados às ações judiciais, a Tabela 3 mostra que: a justiça foi gratuita na maioria $(70,6 \%)$ dos processos; o parecer do Núcleo de Assistência Técnica em Saúde (NATS) estava presente em 23,5\% e o mesmo percentual dos feitos teve perícia judicial; a liminar foi concedida na maioria (70,6\%) dos casos; o tempo entre a distribuição do processo e a liminar não estava presente em quatro ações e, nas outras 13, variou de um a 187 dias, com média de 46,31 dias, desvio padrão de 66,84 dias, mediana de 12,00 dias e percentis 25 e 75 iguais a 3,00 e 67,50 dias, respectivamente.

Tabela 3. Avaliação dos dados relacionados à ação judicial

\begin{tabular}{lc}
\hline Variável & Grupo total \\
\hline TOTAL: $n(\%)$ & $17(100,0)$ \\
\hline Ano do procedimento & $\mathrm{n}(\%)$ \\
\hline 2009 a 2014 & $6(35,3)$ \\
2015 a 2017 & $11(64,7)$ \\
\hline Tipo de processo & $\mathrm{n}(\%)$ \\
\hline Físico & $12(70,6)$ \\
Eletrônico & $5(29,4)$ \\
\hline Comarca & $\mathrm{n}(\%)$ \\
\hline Recife & $14(82,4)$ \\
Caruaru & $3(17,6)$ \\
\hline
\end{tabular}


Alcoforado R. L. G., Almeida A. C., Porto G. G., Silva J. C., Antunes A. A.

Continuação

\begin{tabular}{|c|c|}
\hline Variável & Grupo total \\
\hline Justiça gratuita & $\mathrm{n}(\%)$ \\
\hline Sim & $12(70,6)$ \\
\hline Não & $5(29,4)$ \\
\hline Valor da causa (mil reais) & $\mathrm{n}(\%)$ \\
\hline 5 a 45 & $5(29,4)$ \\
\hline Mais de 45 a 101 & $4(23,5)$ \\
\hline Não informado & $8(47,1)$ \\
\hline Parecer NATS & $\mathrm{n}(\%)$ \\
\hline Sim & $4(23,5)$ \\
\hline Não & $13(76,5)$ \\
\hline Perícia judicial & $\mathrm{n}(\%)$ \\
\hline Sim & $4(23,5)$ \\
\hline Não & $13(76,5)$ \\
\hline Concessão da tutela de urgência & $\mathrm{n}(\%)$ \\
\hline Sim & $12(70,6)$ \\
\hline Não & $5(29,4)$ \\
\hline $\begin{array}{l}\text { Tempo entre distribuição processo e a concessão limiar (dias): } \\
\text { Média } \pm \text { DP (Mediana) }\end{array}$ & $46,31 \pm 66,84(12,00)$ \\
\hline Tempo entre a distribuição do processo e a concessão da liminar (dias) & $\mathrm{n}(\%)$ \\
\hline 1 a 10 & $6(35,3)$ \\
\hline 11 a 20 & $1(5,9)$ \\
\hline 21 a 30 & $1(5,9)$ \\
\hline 31 a 40 & $1(5,9)$ \\
\hline 41 ou mais & $4(23,5)$ \\
\hline Não informado & $4(23,5)$ \\
\hline BacenJud & $\mathrm{n}(\%)$ \\
\hline Sim & $2(11,8)$ \\
\hline Não & $15(88,2)$ \\
\hline Parecer favorável do MP & $\mathrm{n}(\%)$ \\
\hline Sim & $12(70,6)$ \\
\hline Não & $5(29,4)$ \\
\hline Confirmação da liminar pela sentença & $\mathrm{n}(\%)$ \\
\hline Aguardando sentença & $7(41,2)$ \\
\hline Procedente & $9(52,9)$ \\
\hline Improcedente & $1(5,9)$ \\
\hline Recurso & $\mathrm{n}(\%)$ \\
\hline Não & $4(23,5)$ \\
\hline Sim, agravo & $5(29,4)$ \\
\hline Sim, AP & $7(41,2)$ \\
\hline Sim, ED & $1(5,9)$ \\
\hline
\end{tabular}




\begin{tabular}{lc} 
Continuação & \\
\hline Variável & Grupo total \\
\hline Decisão reformada & $\mathrm{n}(\%)$ \\
\hline Aguarda julgamento & $5(29,4)$ \\
Não & $12(70,6)$ \\
\hline Condenação da SASSSEPE DM & $\mathrm{n}(\%)$ \\
\hline Aguardando sentença & $7(41,2)$ \\
Sim & $5(29,4)$ \\
Não & $5(29,4)$ \\
\hline Procedimento realizado & $\mathrm{n}(\%)$ \\
\hline Sim & $8(47,1)$ \\
Não & $9(52,9)$ \\
\hline Trânsito em julgado & $\mathrm{n}(\%)$ \\
\hline Sim & $4(23,5)$ \\
Não & $13(76,5)$ \\
\hline
\end{tabular}

Em relação ao tempo entre a distribuição do processo e a concessão da liminar, a maior frequência foi de até 10 dias (35,3\%); para 23,5\% dos casos, foi superior a 40 dias; e teve frequência unitária para as outras três faixas de tempo consideradas.

A ocorrência de BacenJud (penhora on-line) foi registrada em 11,8\% das ações. A maioria dos processos (70,6\%) teve parecer favorável do Ministério Público. Em 52,9\% a sentença foi considerada procedente; em 5,9\%, improcedente; e os $41,2 \%$ demais estavam aguardando a sentença.

\section{Discussão}

Os direitos fundamentais inerentes à condição humana acarretam a busca pela preservação da saúde em vários aspectos. A saúde bucal, em virtude do avanço das ciências da saúde e do consequente envelhecimento populacional, tornou-se elemento de substancial importância para a sedimentação da qualidade de vida do indivíduo ${ }^{20}$.

O presente estudo buscou mapear as ações judiciais envolvendo o SASSEPE relativas às cirurgias bucomaxilofaciais com uso de OPMEs, no período de janeiro de 2009 a dezembro de 2017, perante a justiça estadual de Pernambuco.

No estudo em pauta, foi possível identificar 17 processos, dos quais $80 \%$ dos autores tinham idade superior a 50 anos e a maioria (média de $82,4 \%$ ) era do sexo

\footnotetext{
${ }^{20}$ MELO, M. Barbosa de. O direito internacional do desenvolvimento e a perspectiva dos países que compõem os BRICs. 2018. Tese (Doutorado) - Faculdade de Direito de Coimbra, 2018. p. 157.
} 
feminino. Em relação ao gênero, pesquisa realizada em 2009 em Florianópolis (SC) com adultos entre 20 e 59 anos apontou perda maior de dentes entre as mulheres ${ }^{21}$, o que pode ajudar a explicar este dado. Acredita-se que o número acentuado de edentulismo no sexo feminino derive de uma maior utilização dos serviços odontológicos, especificamente os de prática curativista, cujo resultado pode ocasionar sobrestamento e perdas dos dentes ${ }^{22}$.

Quanto à idade, já se demonstrou que a saúde bucal e a preservação dos dentes passa a ser um requisito relevante na qualidade de vida do grupo populacional mais velho, devido ao panorama etário mundial de aumento constante da expectativa de vida. De acordo com o Instituto Brasileiro de Geografia e Estatística (IBGE), a projeção para 2050 é de percentual igual de idosos e adolescentes ${ }^{23}$.

$\mathrm{Na}$ atualidade, com esse envelhecimento da população, percebe-se o aumento do uso de próteses por portadores de deformidade bucomaxilofacial, por proporcionarem, dentre outros benefícios, a reparação estética e o aumento da qualidade de $v_{i d a}{ }^{24}$.

Um dos entraves à utilização de OPMEs é o alto custo dos materiais, devido à complexidade da reconstrução das estruturas bucomaxilofaciais, bem como a ausência de cobertura quando se trata de procedimentos meramente estéticos. No caso do SASSEPE, a contribuição média mensal do usuário é de apenas $R \$ 150$ por pessoa, abarcando a assistência à saúde e a bucal. Em contrapartida, segundo a $\mathrm{ANS}^{25}$, o valor comercial médio de um plano ambulatorial e hospitalar praticado em Pernambuco totalizava R $\$ 1.423,59$ (54 a 58 anos) e R $\$ 1.957,86$ (59 anos ou mais) em dezembro de 2017.

A pesquisa demonstrou que $88,3 \%$ dos procedimentos solicitados foram de cirurgias ortognáticas e/ou reconstrutivas, com hipótese diagnóstica mais frequente de deformidade dentoesquelética (52,9\%) e atrofia óssea dos maxilares (29,4\%), e valor médio de $\mathrm{R} \$ 129$ mil para o procedimento.

\footnotetext{
${ }^{21}$ BARBATO, Paulo Roberto et al. Indicadores contextuais e individuais associados à presença de dentes em adultos. Rev. Saúde Pública, São Paulo, v. 49, n. 27, maio 2015. Disponível em: https://www.scielo.br/pdf/rsp/v49/pt_0034-8910-rsp-S0034-89102015049005535.pdf. https://doi.org/10.1590/S0034-8910.2015049005535.

${ }^{22}$ DALAZEN, Chaiane Emilia; BOMFIM, Rafael Aiello; DE-CARLI, Alessandro Diogo. Fatores associados à autopercepção da necessidade de tratamento odontológico e de prótese em idosos brasileiros. Ciênc. saúde coletiva, Rio de Janeiro, v. 23, p. 945-952, 2018. Disponível em https://www.scielo.br/pdf/csc/ v23n3/1413-8123-csc-23-03-0945.pdf. http://dx.doi.org/10.1590/1413-81232018233.09682016.

${ }^{23}$ OLIVEIRA, Avilene Marta de. 0 envelhecimento e a Odontologia. Memorialidades, v. 2, n. 3 e 4, p. 9-14, 2014. Disponível em: https://periodicos.uesc.br/index.php/memorialidades/article/view/174/182.

${ }^{24}$ LIMA, Cacilda Chaves Morais de. Impacto da reabilitação bucomaxilofacial sobre o estado nutricional, sintomas depressivos, autoimagem, autoestima e qualidade de vida em adultos e idosos. 2014. Tese (Doutorado) - Programa de Pós-Graduação em Gerontologia Biomédica, Pontifícia Universidade Católica do Rio Grande do Sul, Porto Alegre, 2014.

${ }^{25}$ PAINEL de precificação: planos de saúde. Disponível em: http://biblioteca-ans/index.php?codigo_ sophia=7005. Acesso em: 23 jul. 2018.
} 
O uso de dispositivos implantáveis é bastante dispendioso, razão pela qual sua indicação deve ser bem criteriosa. O mercado nacional de produtos médicos movimentou R \$ 19,7 bilhões em 2014, dos quais R \$ 4 bilhões (cerca de 20\%) foram com dispositivos médicos implantáveis (DMIs). O maior faturamento no setor de saúde no Brasil referia-se aos equipamentos, mas a categoria DMI foi a que teve a maior taxa de crescimento, de $249 \%$ entre 2007 e $2014^{26}$. Pesquisa realizada em 2012 pela ANS junto às cinco maiores operadoras de planos privados de saúde em cada modalidade evidenciou que cerca de $10 \%$ do total das despesas assistenciais referiam-se a despesas com $\mathrm{OPME}^{27}$. Nesse diapasão, os consumidores são os maiores prejudicados, tendo em vista que, com a crescente demanda por medicamentos e materiais de alto custo, as mensalidades dos planos de saúde ficam cada vez mais dispendiosas.

Ciente de tal cenário, a ANS, por meio do Parecer Técnico n. 24/2019²8, disciplina que OPMEs e DMIs devem ser previamente registrados na Anvisa para que possam ser fornecidos pelos planos privados de assistência à saúde.

Em relação às demandas contra o SASSEPE analisadas, constatou-se que em 76,5\% não havia cobertura contratual para os OPMEs solicitados pelos autores, seja por falta de registro junto a Anvisa, seja pelo caráter estético ou experimental do procedimento.

A atual crise econômica do país e seu impacto na saúde demandam a adoção de ações que convirjam para a eficiência e o combate de gastos desnecessários, em especial os que decorram de ações ilegais. Essas novas práticas ocorrem em benefício da sociedade e, sobretudo, daqueles que atuam de maneira ética, com conhecimento técnico para enfrentar os desafios e as resistências ${ }^{29}$.

Constatou-se que a cirurgia ortognática vem sendo utilizada com frequência para tratar deformidades dentofaciais, tendo em vista que os procedimentos cirúrgicos podem afetar tanto a aparência facial como o espaço aéreo posterior ${ }^{30}$. Dentre outros benefícios, a grande maioria dos pacientes recorre a esse tipo de

\footnotetext{
${ }^{26}$ GRUPO de Trabalho Interinstitucional Sobre Órteses, Próteses e Materiais Especiais (GTI-OPME). Relatório Final do Grupo de Trabalho Interinstitucional sobre órteses, próteses e materiais especiais (GTI-OPME). Brasília-DF, 2015. Disponível em: http://portalarquivos.saude.gov.br/images/pdf/2015/julho/07/Relato rio-Final-versao-final-6-7-2015.pdf.

${ }^{27}$ AGÊNCIA NACIONAL DE SAÚDE SUPLEMENTAR - ANS. Pesquisa de preço sobre Órteses, Próteses e Materiais Especiais (OPME). 2012.

${ }^{28}$ AGÊNCIA NACIONAL DE SAÚDE SUPLEMENTAR - ANS. Parecer Técnico n² 24/GEAS/GGRAS/DIPRO/2019. Cobertura: Órteses, Próteses e Materiais Especiais - OPME. Disponível em http://www.ans.gov.br/images/ stories/parecer_tecnico/uploads/parecer_tecnico/_parecer_2019_24.pdf.

${ }^{29}$ GRUPO de Trabalho Externo de Órteses, Próteses, e Materiais Especiais (GTE OPME) - ANS/ ANVISA. Relatório Final. Disponivel em: http://antigo.anvisa.gov.br/documents/219201/2782895/Relat\%C3\%B3rio_ OPME_compilado_low.pdf/bfcbbcde-7be3-4f87-91b0-37ee5a27e777. Acesso em: 16 fev. 2021.

${ }^{30}$ PORTO, Bruna Pires. Avaliação de medidas de área de vias aéreas superiores em radiografias de pacientes submetidos a cirurgia ortognática. 2018. Monografia (Graduação) - Faculdade de Odontologia, Universidade Federal do Rio Grande do Sul, Porto Alegre, 2018.
} 
cirurgia devido à insatisfação com a estética dentofacial, na busca por uma suposta melhora na aparência dentária e facial ${ }^{31}$.

Causa preocupação o fato de o Poder Judiciário, em sede de tutela antecipada, determinar o fornecimento de procedimentos não previstos nos contratos e, em alguns casos, até sem registro nas agências reguladoras. Insta mencionar que a tutela de urgência foi concedida em $70,6 \%$ dos processos analisados e que houve parecer do NATS em apenas $23,5 \%$.

O sistema público de saúde e os planos privados não podem fornecer todo e qualquer tratamento ou material solicitado pelos usuários e beneficiários. Além da previsão e regulamentação pelo ordenamento jurídico, é imprescindível o uso sistemático de evidências científicas para a concessão de liminares em saúde.

O Ministério da Saúde ${ }^{32}$ entende que a pouca utilização das evidências científicas por parte do Judiciário deve-se a diferentes fatores, entre eles dificuldade que os tomadores de decisão têm de aplicar os conhecimentos científicos.

Outro fato destacado no resultado foi que, apesar de o SASSEPE possuir um centro de saúde bucal, 94,1\% dos autores foram assistidos por profissionais não credenciados, os quais, em sua totalidade, indicaram marca ou fornecedor específico e optaram pela realização do procedimento em consultório $(41,2 \%)$. O problema quanto à indicação de OPMEs de marcas exclusivas reflete um obstáculo vivenciado nos serviços de saúde em geral, tanto os privados quanto os públicos. Apesar de a legislação estipular que ao cirurgião-dentista é vedada a indicação de marca comercial quando da solicitação de próteses, órteses ou materiais especiais, a realidade vem se mostrando outra ${ }^{33}$. Muitos profissionais não seguem a Resolução n. 115/2012 ${ }^{34}$ do CFO ao não indicarem o nome de três tipos de materiais, fornecidos por empresas diferentes, com os quais gostariam de trabalhar. Entende-se que seja natural fazer a escolha do material pela qualidade do produto e pela afinidade do cirurgião, mas não se pode esquecer a determinação legal de oferecer, pelo menos, três tipos distintos. O direcionamento a algum fornecedor poderá impossibilitar a compra do OPME pelo melhor preço, tanto pela operadora de saúde como pelo ente público.

\footnotetext{
${ }^{31}$ FABER, Jorge; FABER, Ana Paula Megale Hecksher. Percepções das deformidades dentofaciais: do bem-estar psíquico à indicação de cirurgia. Dental Press J. Orthod, v. 15, n. 4, p. 13-14, 2010. Disponível em: https:// www.scielo.br/pdf/dpjo/v15n4/02.pdf. http://dx.doi.org/10.1590/S2176-94512010000400002.

${ }^{32}$ MINISTÉRIO DA SAÚDE - MS. Secretaria de Ciência, Tecnologia e Insumos Estratégicos. Departamento de Ciência e Tecnologia. Síntese de evidências para políticas de saúde: estimulando o uso de evidências científicas na tomada de decisão. Brasília-DF: Ministério da Saúde; EVIPNet Brasil, 2015. 36 p. Disponível em https://bvsms.saude.gov.br/bvs/publicacoes/sintese_evidencias_politicas_saude_1ed.pdf.

${ }^{33}$ MARTINS, Paulo Roberto do Nascimento; DAHINTEN, Bernardo Franke; DAHINTEN, Augusto Franke. op. cit., p. 145-166.

${ }^{34}$ CONSELHO FEDERAL DE ODONTOLOGIA - CFO. Resolução CFO-115, de 03 de abril de 2012. Disciplina a prescrição de materiais de implante, órteses e próteses, e determina arbitragem de conflitos. Disponível em: http://transparencia.cfo.org.br/ato-normativo/?id=1630. Acesso em: 24 jul. 2018.
} 
De outro giro, instaurou-se, em regra, uma verdadeira lide entre o cirurgião indicado pela parte autora e as operadoras de saúde, as quais visam a diminuir seus custos com a observância de algumas práticas: análise prévia à internação e requisição do uso de OPME; negociação de valores de materiais e taxas de comercialização com os hospitais; negociação e compra de materiais diretamente com os fornecedores; remuneração de cirurgias por pacotes, com os custos de OPMEs inclusos no valor; estabelecimento de protocolos de autorização e precificação dos materiais, negociação com o prestador de serviços; convite aos pacientes a se submeterem a consulta de segunda opinião $0^{35}$.

O tema é de tamanha envergadura, que não passou despercebido pelo legislador. Tramita desde fevereiro de 2015 no Congresso Nacional o Projeto de Lei do Senado n. 017/2015, que pretende introduzir mecanismos de controle e regulação dos preços de OPMEs, com previsão de multas vultuosas para quaisquer empresas ou pessoas que pagarem ou receberem comissão em razão da prescrição desses materiais ${ }^{36}$.

O SASSEPE implantou em 2016 o mecanismo de "segunda opinião médica", com o objetivo de reduzir gastos exorbitantes com OPMEs, evitando procedimentos desnecessários ${ }^{37}$. No atual cenário da saúde suplementar, o papel do profissional auditor tornou-se um elemento imprescindível para avaliação e controle dos procedimentos e materiais solicitados, ao assessorar os gestores em questões absolutamente técnicas.

$\mathrm{Na}$ verdade, esse novo mecanismo funciona como uma espécie de perícia administrativa, para que um técnico possa analisar o procedimento e o material solicitados. Constatou-se que tal análise foi realizada em 52,9\% dos casos estudados. Nos demais casos, acredita-se que não tenha havido tempo hábil para a segunda opinião médica, tendo em vista a usual celeridade da justiça na concessão da tutela de urgência, especificamente em matéria de saúde -a presente pesquisa observou que o tempo médio entre a distribuição do processo e a concessão da liminar foi de apenas 12 dias.

Verificou-se que a tutela foi concedida sem a ouvida da parte contrária em $70,6 \%$ dos casos estudados e que, em $52,9 \%$ deles, foi confirmada na sentença, com parecer favorável do Ministério Público em 70,6\% dos feitos. Em recente estudo ${ }^{38}$,

\footnotetext{
${ }^{35}$ ALENCAR, Anna Carolyne Ferreira. op. cit.

${ }^{36}$ SENADO FEDERAL. Projeto de Lei n. 17/2015. Disponível em: https://www25.senado.leg.br/web/ atividade/materias/-/materia/119638. Acesso em: 14 ago. 2018.

${ }^{37}$ CONSELHO DELIBERATIVO DO SISTEMA DE ASSISTÊNCIA À SAÚDE DOS SERVIDORES DO ESTADO DE PERNAMBUCO -- CONDASPE. Resolução n. 01/2016. Instituir o mecanismo de "Segunda Opinião Médica" no âmbito do Sistema de Assistência à Saúde dos Servidores do Estado de Pernambuco - SASSEPE, na forma prevista no Art. $2^{\circ}$ e $7^{\circ}$ da Lei complementar $n^{\circ} 30$ de 02 de janeiro de 2001. Disponível em: http:// www.portais.pe.gov.br/c/document_library/get_file?uuid=ad172229-4ada-4c31-91b9-559827174702\&g roupld=19517. Acesso em: 16 fev. 2021.

${ }^{38}$ OLIVEIRA, Fábio Henrique Cavalcanti de et al. Judicialização do Acesso aos Serviços de Saúde: análise de caso da Secretaria de Saúde de Pernambuco. Revista Cadernos Ibero-Americanos de Direito Sanitário, v. 7, n. 2, p.173-186, 2018. Disponível em: https://www.cadernos.prodisa.fiocruz.br/index.php/cadernos/ article/view/489/544. https://doi.org/10.17566/ciads.v7i2.489.
} 
constatou-se que, das 2.560 ações judiciais ajuizadas em 2016 contra o estado de Pernambuco envolvendo questão de saúde, em 2.438 (95,2\%) foram deferidas liminares e em apenas 115 (4,5\%) o pedido foi negado.

Ao dissecar as decisões acima mencionadas, vê-se que o principal argumento utilizado para deferir os pedidos dos autores foi o direito constitucional à saúde $\left(\mathrm{CF} / 88\right.$, arts. 196 e 199) e o direito do consumidor ${ }^{39}$. Em contrapartida, o Superior Tribunal de Justiça (STJ) ${ }^{40}$ editou no dia 11 de abril de 2018 a Súmula n. 608, excluindo a incidência do Código de Defesa do Consumidor nos casos envolvendo os planos de autogestão, como o SASSEPE: "Aplica-se o Código de Defesa do Consumidor aos contratos de saúde, salvo os administrados por entidades de autogestão".

As súmulas concentram o entendimento de uma série de precedentes do mesmo tribunal. Embora os magistrados não sejam obrigados a adotá-las, costumam seguir suas orientações. Dessa forma, acredita-se que o número de ações contra o SASSEPE sofrerá uma diminuição, tendo em vista ser um plano de autogestão, sem finalidade lucrativa e exclusivo dos servidores públicos do estado de Pernambuco.

Outro dado importante refere-se à concessão do benefício da justiça gratuita, observado em $70,6 \%$ dos casos. Assim, caso o autor tenha seus pedidos julgados improcedentes, estará dispensado de pagar os honorários (advocatícios sucumbenciais e periciais), nos termos da lei ${ }^{41}$. Embora essa questão não integre o objetivo específico da pesquisa, insta mencionar que, apesar de existirem correntes sobre suposta abusividade na concessão da gratuidade, faltam dados concretos sobre sua verificação ${ }^{42}$.

Em todos os casos analisados, constatou-se que não havia urgência por parte dos autores que justificasse a busca por uma rápida resposta judicial. Ninguém estava correndo risco de vida ou de um dano supostamente irreversível em curto prazo. Por outro lado, existem casos em que é plenamente possível a concessão de liminar, sem a prévia formação do contraditório, como o fornecimento de medicamentos e insumos essenciais para a manutenção da vida.

Observou-se que, nos casos analisados, não deveria o julgador ter proferido a tutela de urgência ${ }^{43}$ liminarmente - isto é, no início do processo - sem a prévia

\footnotetext{
${ }^{39}$ BRASIL. Lei n. 8.078, de 11 de setembro de 1990. Dispõe sobre a proteção do consumidor e dá outras providências. Disponível em: http://www.planalto.gov.br/ccivil_03/leis/I8078compilado.htm. Acesso em: 16 fev. 2021.

${ }^{40}$ SUPERIOR TRIBUNAL DE JUSTIÇA - STJ. Súmula n. 608. Aplica-se o Código de Defesa do Consumidor aos contratos de plano de saúde, salvo os administrados por entidades de autogestão. (Súmula 608, SEGUNDA SEÇÃO, julgado em 11/04/2018, DJe 17/04/2018). Disponível em: https://scon.stj.jus.br/SCON/sumanot/ toc.jsp?livre=(sumula\%20adj1\%20\%27608\%27).sub.\#TIT1TEMAO. Acesso em: 16 fev. 2021.

${ }^{41}$ BRASIL. Lei n. 1.060, de 5 de fevereiro de 1950. Estabelece normas para a concessão de assistência judiciária aos necessitados. Disponivel em: http://www.planalto.gov.br/ccivil_03/leis/l1060.htm. Acesso em: 16 fev. 2021.

${ }^{42}$ TARTUCE, Fernanda; DELLORE, Luiz. Gratuidade da justiça no novo CPC. Revista de Processo, São Paulo, v. 39, n. 236, p. 305-323, out. 2014.

${ }^{43}$ BRASIL. Lei n. 13.105, de 16 de março de 2015. Código de Processo Civil. Disponível em: http://www. planalto.gov.br/ccivil_03/_ato2015-2018/2015/lei/I13105.htm. Acesso em: 16 fev. 2021.
} 
oitiva da Procuradoria do Estado, a consulta ao NATS $^{44}$ e até mesmo a realização da prova pericial.

Em Pernambuco, o NATS foi criado após a Recomendação n. 31/2010 45 do CNJ e consiste em uma parceria entre o Tribunal de Justiça de Pernambuco e o governo do Estado de Pernambuco. É integrado por uma equipe composta por uma médica, uma advogada e uma farmacêutica, com a finalidade de assessorar os magistrados no que tange às demandas judiciais da saúde. Devido ao sucesso dessa parceria, o Tribunal Regional Federal da $5^{\text {a }}$ Região (TRF5) assinou um termo de cooperação com o Tribunal de Justiça de Pernambuco para auxiliar os juízes federais na apreciação de liminares envolvendo questões de saúde.

Verificou-se, curiosamente, que somente em 23,5\% dos casos analisados houve parecer do NATS do Tribunal de Justiça de Pernambuco e produção da perícia judicial. Ora, decidir sobre a vida alheia é um ofício de responsabilidade infinita, razão pela qual o magistrado deve fundamentar sua decisão com base técnica e jurídica (CF/88, art. 93, X), além de manter a imparcialidade, para não se deixar influenciar pelo calor da emoção gerada pelos argumentos da parte autora. Sobre o tema, aduz Marcos Antônio da Costa Sabino quando dispõe sobre a denominada justiça de misericórdia:

Este autor já ouviu de magistrados que, diante de um pedido individual por certo medicamento, a concessão será sempre medida de rigor, porque, caso contrário, eles, juízes, não conseguiriam se beneficiar do tranquilo sono dos justos. Faz sentido, então, que os pacientes aleguem urgência, risco de morte e contem histórias bem tristes, como forma de convencer os julgadores. Mas o argumento emocional não é justificativa hábil a explicar ou fundamentar as decisões em casos individuais, sobretudo sob o viés técnico. E, com efeito, a justiça de misericórdia é um grave problema da concessão judicial de acesso à saúde ${ }^{46}$.

Além do viés emocional, acredita-se que a grande maioria dos magistrados não tenha conhecimentos técnicos em saúde, principalmente no que tange a OPMEs utilizados em cirurgias bucomaxilofaciais. Assim, torna-se necessária uma maior estruturação do NATS pelos tribunais, inclusive com a presença de um cirurgião bucomaxilofacial, com a finalidade de auxiliar os julgadores nessa árdua missão de julgar e de buscar o ideal de justiça. Sobre o assunto, escreve Francisco Glauber Pessoa Alves:

\footnotetext{
${ }^{44}$ PARCERIA entre o TJPE e o Poder Executivo auxilia magistrados em ações judiciais relacionadas à saúde. Assessoria de Comunicação do Tribunal de Justiça de Pernambuco, 03 mar. 2015. Disponível em: https:// www.tjpe.jus.br/intranet/noticias_ascomSY/ver_noticia.asp?id=9762. Acesso em: 30 jul. 2018.

${ }^{45}$ CONSELHO NACIONAL DE JUSTIÇA - CNJ. Resolução n. 31, de 30 de março de 2010. Disponível em http:// www.cnj.jus.br/atos-normativos?documento=877. Acesso em: 16 fev. 2021.

${ }^{46}$ SABINO, Marco Antonio da Costa. Saúde e Judiciário: a atuação judicial-limites, excessos e remédios. Curitiba: Juruá, 2016. p. 138.
} 
O julgador não pode ser ingênuo: há forte lobby de fabricantes e laboratórios farmacêuticos junto a médicos para prescrição de seus medicamentos e produtos. Essas relações eticamente discutíveis existem e não podem ser ignoradas, donde a importância de instrução mínima (manifestação do NAT, oitiva do gestor do SUS e nomeação de perito judicial) a sindicar e confirmar necessidades especiais (fármacos, tratamentos, procedimentos, suplementos) e mais custosas prescritas pelo médico particular da parte ${ }^{47}$.

Ademais, não se pode esquecer a relevância da perícia técnica judicial, produzida quando ocorre a necessidade de um conhecimento técnico determinado para se definirem os contornos dos fatos. Tal prova deve ser elaborada por um perito nomeado pelo juiz, de sua extrema confiança, para análise das questões técnicas e dos argumentos levantados pelos litigantes ${ }^{48}$.

\section{Considerações finais}

Em uma sociedade movida pelos ideais de beleza, é cada vez mais raro o uso de dentaduras e da simples extração dos dentes sem o uso de algum tipo de OPME. Assim, com o aumento da expectativa de vida e o avanço da odontogeriatria, as próteses bucomaxilofaciais passaram a proporcionar, dentre outros benefícios, a reparação estética. Um dos entraves ao uso desses materiais é seu alto custo, que se deve à complexidade da reconstrução das estruturas bucomaxilofaciais, bem como a ausência de cobertura de procedimentos meramente estéticos. Tais óbices acarretam a judicialização.

A análise dos processos demonstrou que, em regra, não estavam presentes os requisitos para a concessão da tutela de urgência. Nesses casos, o ideal seria ter havido uma prévia manifestação do NATS ou da Procuradoria do Estado.

Verificou-se, ainda, a necessidade de maior transparência sobre a imprescindibilidade do procedimento solicitado, até como forma de minimizar as chances de práticas antiéticas e ilegais. Assim, espera-se que o Congresso Nacional aprove o projeto de Lei do Senado n. 017/2015, com a finalidade de pelo menos mitigar eventuais conluios entre fabricantes e distribuidores de OPMEs.

Tendo como premissa a importância constitucional dada ao direito à saúde, apurou-se uma necessidade de maior investimento na formação e no

\footnotetext{
${ }^{47}$ ALVES, Francisco Glauber Pessoa. Ações de saúde contra o poder público: ensaio de um roteiro decisório. Revista Jurídica da Seção Judiciária de Pernambuco, n. 9, p. 55-97, 2017. Disponível em: https://revista. jfpe.jus.br/index.php/RJSJPE/article/download/148/139.

${ }^{48}$ KALLAS FILHO, Elias; FONSECA, João Paulo de Oliveira. A influência da prova pericial nas decisões judiciais acerca da responsabilidade civil dos médicos. Revista de Direito Sanitário, São Paulo, v. 16, n. 2, p. 101-115, 2015. Disponível em: https://www.revistas.usp.br/rdisan/article/view/106885/105505. http:// dx.doi.org/10.11606/issn.2316-9044.v16i2p101-115.
} 
aperfeiçoamento não apenas dos magistrados, mas de todo o Poder Judiciário, com a finalidade de subsidiar os julgadores e assegurar uma maior eficiência na solução das demandas envolvendo a assistência à saúde. Assim, espera-se que a judicialização possa ser considerada uma ferramenta robusta para tal finalidade.

\section{Referências}

ABADE, Erik Asley Ferreira. Judicialização da assistência médica suplementar: um estudo de caso em Pernambuco. 2015. Dissertação (Mestrado). Programa de Pós-Graduação em Saúde Coletiva, Universidade Federal da Bahia, ISC-UFBA, Salvador-BA, 2015. Disponível em: http://repositorio.ufba.br/ri/handle/ri/1791. Acesso em: 08 jun. 2018.

AGÊNCIA NACIONAL DE SAÚDE SUPLEMENTAR - ANS. Parecer Técnico n 24/ GEAS/GGRAS/DIPRO/2019. Cobertura: Órteses, Próteses e Materiais Especiais - OPME. Disponível em http://www.ans.gov.br/images/stories/parecer_tecnico/uploads/parecer_ tecnico/_parecer_2019_24.pdf.

AGÊNCIA NACIONAL DE SAÚDE SUPLEMENTAR - ANS. Pesquisa de preço sobre Órteses, Próteses e Materiais Especiais (OPME). 2012.

ALENCAR, Anna Carolyne Ferreira. Aquisição e utilização das órteses, próteses e materiais especiais-OPME e os facilitadores do superfaturamento no sistema de saúde. 2016. Monografia (Bacharelado em Gestão em Saúde Coletiva) - Universidade de Brasília, Brasília-DF, 2016.

ALVES, Francisco Glauber Pessoa. Ações de saúde contra o poder público: ensaio de um roteiro decisório. Revista Jurídica da Seção Judiciária de Pernambuco, n. 9, p. 55-97, 2017. Disponível em: https://revista.jfpe.jus.br/index.php/RJSJPE/article/download/148/139.

ANS disponibiliza números de abril do setor de planos de saúde. Notícias ANS, 05 jun. 2019. Disponível em: http://www.ans.gov.br/aans/noticias-ans/numeros-do-setor/5009-ans-disponi biliza-numeros-de-abril-do-setor-de-planos-de-saude. Acesso em: 07 jun. 2019.

ASENSI. Felipe Dutra; PINHEIRO Roseni. Judicialização da saúde no Brasil: dados e experiência. Brasília-DF: Conselho Nacional de Justiça, 2015. Disponível em: http://www. cnj.jus.br/files/conteudo/destaques/arquivo/2015/06/6781486daef02bc6ec8c1e491a565006. pdf. Acesso em: 30 jul. 2018.

BARBATO, Paulo Roberto et al. Indicadores contextuais e individuais associados à presença de dentes em adultos. Rev. Saúde Pública, São Paulo, v. 49, n. 27, maio 2015. Disponível em: https://www.scielo.br/pdf/rsp/v49/pt_0034-8910-rsp-S0034-89102015049005535.pdf. https://doi.org/10.1590/S0034-8910.2015049005535.

BARBATO, Paulo Roberto et al. Indicadores contextuais e individuais associados à presença de dentes em adultos. Rev. Saúde Pública, São Paulo, v. 49, maio 2015. Disponível em: https://www.scielo.br/pdf/rsp/v49/pt_0034-8910-rsp-S0034-89102015049005535.pdf. https://doi.org/10.1590/S0034-8910.2015049005535. 
Alcoforado R. L. G., Almeida A. C., Porto G. G., Silva J. C., Antunes A. A.

COLÉGIO BRASILEIRO DE CIRURGIA E TRAUMATOLOGIA BUCO-MAXILO-FACIAL. Parâmetros e recomendações para procedimentos buco-maxilo-faciais do Colégio Brasileiro de Cirurgia e Traumatologia Buco-Maxilo-Facial. Ênfase em OPME. Versão 2017/2018. Edição Compacta. Disponível em: https://www.bucomaxilo.org.br/upfiles/downloads/diretrizes-e-re comendacoes-para-procedimentos-bmf.pdf. Acesso em: 16 fev. 2021.

CONSELHO NACIONAL DE JUSTIÇA - CNJ. Relatório analítico propositivo sobre a judicialização da saúde no Brasil. Brasília-DF: CNJ, maio, 2019. Disponível em: http://www. cnj.jus.br/files/conteudo/arquivo/2019/03/f74c66d46cfea933bf22005ca50ec915.pdf. Acesso em: 27 maio 2019.

DALAZEN, Chaiane Emilia; BOMFIM, Rafael Aiello; DE-CARLI, Alessandro Diogo. Fatores associados à autopercepção da necessidade de tratamento odontológico e de prótese em idosos brasileiros. Ciênc. saúde coletiva, Rio de Janeiro, v. 23, p. 945-952, 2018. Disponível em https://www.scielo.br/pdf/csc/v23n3/1413-8123-csc-23-03-0945.pdf. http://dx.doi. org/10.1590/1413-81232018233.09682016.

FABER, Jorge; FABER, Ana Paula Megale Hecksher. Percepções das deformidades dentofaciais: do bem-estar psíquico à indicação de cirurgia. Dental Press J. Orthod, v. 15, n. 4, p. 13-14, 2010. Disponível em: https:/www.scielo.br/pdf/dpjo/v15n4/02.pdf. http://dx.doi.org/10.1590/ S2176-94512010000400002.

FUNDAÇÃO GETÚlIO VARGAS - FGV. 2018. FGV e Instituto Brasileiro de Econômica. Maio 2019. Disponível em https://portalibre.fgv.br/data/files/30/F2/67/E0/ EE4EA61078ADFDA68904CBA8/BoletimMacroIbre_1905.pdf. Acesso em: 07 jun. 2019.

GRUPO de Trabalho Externo de Órteses, Próteses, e Materiais Especiais (GTE OPME) - ANS/ ANVISA. Relatório Final. Disponível em: http://antigo.anvisa.gov.br/ documents/219201/2782895/Relat\%C3\%B3rio_OPME_compilado_low.pdf/bfcbbcde7be3-4f87-91b0-37ee5a27e777. Acesso em: 16 fev. 2021.

GRUPO de Trabalho Interinstitucional Sobre Órteses, Próteses e Materiais Especiais (GTI-OPME). Relatório Final do Grupo de Trabalho Interinstitucional sobre órteses, próteses e materiais especiais (GTI-OPME). Brasília-DF, 2015. Disponível em: http://portalarquivos. saude.gov.br/images/pdf/2015/julho/07/Relatorio-Final-versao-final-6-7-2015.pdf.

INSTITUTO DE RECURSOS HUMANOS DE PERNAMBUCO - IRH. Sistema de Assistência à Saúde dos Servidores Públicos do Estado de Pernambuco. Disponível em http://www.irh. pe.gov.br/web/irh/sassepe. Acesso em: 10 jun. 2017.

KALLAS FILHO, Elias; FONSECA, João Paulo de Oliveira. A influência da prova pericial nas decisões judiciais acerca da responsabilidade civil dos médicos. Revista de Direito Sanitário, São Paulo, v. 16, n. 2, p. 101-115, 2015. Disponível em: https://www.revistas.usp.br/rdisan/ article/view/106885/105505. http://dx.doi.org/10.11606/issn.2316-9044.v16i2p101-115.

LAUREANO, José Rodrigues et al. Alterações em discrepância ântero-posterior na cirurgia ortognática. Rev. Cir. Traumatol. Buco-Maxilo-Fac. Camaragibe, v. 5, n. 1, p. 45-52, jan./mar. 2005. Disponível em: https://www.revistacirurgiabmf.com/2005/v5n1/pdf\%20v5n1/artigo\%2007.pdf. 
LIMA, Cacilda Chaves Morais de. Impacto da reabilitação bucomaxilofacial sobre o estado nutricional, sintomas depressivos, autoimagem, autoestima e qualidade de vida em adultos $e$ idosos. 2014. Tese (Doutorado) - Programa de Pós-Graduação em Gerontologia Biomédica, Pontifícia Universidade Católica do Rio Grande do Sul, Porto Alegre, 2014.

LIMA, Júlio César França. Neoliberalismo e formação profissional em saúde. Revista Trabalho Necessário, v. 4, n. 4, jun. 2018. Disponível em: https://periodicos.uff.br/trabalhonecessario/ article/view/4613/4249. https://doi.org/10.22409/tn.5i5.p4613.

MÁFIA das próteses coloca vidas em risco com cirurgias desnecessárias. G1, 04 jan. 2015. Disponível em: http://g1.globo.com/fantastico/noticia/2015/01/mafia-das-proteses-colocavidas-em-risco-com-cirurgias-desnecessarias.html. Acesso em: 10 jun. 2017.

MARTINS, Paulo Roberto do Nascimento; DAHINTEN, Bernardo Franke; DAHINTEN, Augusto Franke. O problema envolvendo as OPMES e os planos de saúde: contornos e análise da problemática. Revista de Direito Sanitário, São Paulo, v. 17, n. 1, p. 145-166, 2016. Disponível em: https://www.revistas.usp.br/rdisan/article/view/117052/114650. https://doi.org/10.11606/issn.2316-9044.v17ilp145-166.

MELO, M. Barbosa de. O direito internacional do desenvolvimento e a perspectiva dos países que compóem os BRICs. 2018. Tese (Doutorado) - Faculdade de Direito de Coimbra, 2018.

MINISTÉRIO DA SAÚDE - MS. Secretaria de Ciência, Tecnologia e Insumos Estratégicos. Departamento de Ciência e Tecnologia. Síntese de evidências para políticas de saúde: estimulando o uso de evidências científicas na tomada de decisão. Brasília-DF: Ministério da Saúde; EVIPNet Brasil, 2015.36 p. Disponível em https://bvsms.saude.gov.br/bvs/publicacoes/ sintese_evidencias_politicas_saude_1ed.pdf.

MORAES, Thays Martins de et al. Enxerto de tecido conjuntivo subepitelial para aumento de rebordo: relato de caso. Revista Saúde \& Ciência Online, v. 5, n. 1, p. 91-98, 2016. Disponível em: https://rsc.revistas.ufcg.edu.br/index.php/rsc/article/download/206/202.

ODONTOLOGIA estética cresce 300\% nos últimos três anos. Portal Terra, 07 maio 2018. Disponível em: https://www.terra.com.br/noticias/dino/odontologia-estetica-cresce-300-nosultimos-tres-anos,e141ba60e231a747839a2eaa984f910b9p1r4z10.html. Acesso em 31 jul. 2018.

OLIVA, Juliana Marinho. A constitucionalização do direito civil e a sustentabilidade econômica dos planos de saúde: o caso do Unafisco saúde. 2015. Monografia (Graduação) - Centro Universitário de Brasília, Brasília-DF, 2015.

OLIVEIRA, Avilene Marta de. O envelhecimento e a Odontologia. Memorialidades, v. 2, n. 3 e 4, p. 9-14, 2014. Disponível em: https://periodicos.uesc.br/index.php/memorialidades/ article/view/174/182.

OLIVEIRA, Fábio Henrique Cavalcanti de et al. Judicialização do Acesso aos Serviços de Saúde: análise de caso da Secretaria de Saúde de Pernambuco. Revista Cadernos Ibero-Americanos de Direito Sanitário, v. 7, n. 2, p.173-186, 2018. Disponível em: https://www.cadernos.prodisa. fiocruz.br/index.php/cadernos/article/view/489/544. https://doi.org/10.17566/ciads.v7i2.489. 
PAINEL de precificação: planos de saúde. Disponível em: http://biblioteca-ans/index. php?codigo_sophia=7005. Acesso em: 23 jul. 2018.

PARCERIA entre o TJPE e o Poder Executivo auxilia magistrados em ações judiciais relacionadas à saúde. Assessoria de Comunicação do Tribunal de Justiça de Pernambuco, 03 mar. 2015. Disponível em: https://www.tjpe.jus.br/intranet/noticias_ascomSY/ver_noticia. asp?id=9762. Acesso em: 30 jul. 2018.

PORTO, Bruna Pires. Avaliação de medidas de área de vias aéreas superiores em radiografias de pacientes submetidos a cirurgia ortognática. 2018. Monografia (Graduação) - Faculdade de Odontologia, Universidade Federal do Rio Grande do Sul, Porto Alegre, 2018.

RIBEIRO, Krishina Day; VIDAL, Josep Pont. Uma análise da produção acadêmica sobre a evolução do fenômeno da judicialização da saúde no Brasil. Cadernos Ibero-Americanos de Direito Sanitário, v.7, n.2, p.239-261, abr./jun. 2018. Disponível em: https://www.cadernos. prodisa.fiocruz.br/index.php/cadernos/article/view/493/548. http://dx.doi.org/10.17566/ ciads.v7i2.493.

SABINO, Marco Antonio da Costa. Saúde e Judiciário: a atuação judicial-limites, excessos e remédios. Curitiba: Juruá, 2016.

SCHEFFER, Mário. Coberturas assistenciais negadas pelos planos e seguros de saúde em ações julgadas pelo Tribunal de Justiça do Estado de São Paulo. Revista de Direito Sanitário, São Paulo, v. 14, n. 1, p. 122-131, 2013. Disponível em: https:/www.revistas.usp.br/rdisan/ article/view/56627/59644. https://doi.org/10.11606/issn.2316-9044.v14i1p122-131.

SOUZA, Júlio César de; GOMES, Magno Federici. A judicialização na saúde e a fronteira entre o individual e o coletivo: considerações sobre o acesso ao Sistema Único de Saúde sustentável. Revista Direitos Fundamentais \& Democracia, v. 24, n. 1, p. 216-242, 2019. Disponível em: https://revistaeletronicardfd.unibrasil.com.br/index.php/rdfd/article/view/1227/579. https://doi.org/10.25192/issn.1982-0496.rdfd.v24i11227.

TARTUCE, Fernanda; DELLORE, Luiz. Gratuidade da justiça no novo CPC. Revista de Processo, São Paulo, v. 39, n. 236, p. 305-323, out. 2014.

UCHOA, Severina Alice da Costa; COSTA, Isabelle Katherinne Fernandes; DANTAS, Rodrigo Assis Neves. Os sentidos da integralidade da assistência à saúde: uma revisão da literatura. InterSciencePlace, v. 1, n. 12, p. 78-91, 2015. Disponível em: http://www.interscienceplace. org/isp/index.php/isp/article/download/121/120. 
Renato Ludmer Guedes Alcoforado - Mestrando em Perícias Forenses pela Universidade de Pernambuco(UPE); especialização em Direitos Humanos pela Universidade Católica de Pernambuco (Unicap); graduação em Direito pela Unicap. Recife/PE, Brasil. E-mail: renatoludmer@upe.br

Adriana Conrado de Almeida - Doutorado em Saúde Materno-Infantil pelo Instituto de Medicina Integral Professor Fernando Figueira. Docente da Universidade de Pernambuco (UPE). Recife/PE, Brasil. E-mail: aconradoalmeida@yahoo.com.br

Gabriela Granja Porto - Doutora em Odontologia pela Universidade de Pernambuco (UPE). Professora Adjunta do Programa de Mestrado em Perícias Forenses da UPE. Recife/PE, Brasil. E-mail: gabriela.porto@upe.br

Jackellyne Carneiro da Silva - Mestranda em Perícias Forenses pela Universidade de Pernambuco (UPE); especialização em Enfermagem do Trabalho e em Auditoria da Saúde pela Faculdade Redentor; graduação em Enfermagem pela Fundação de Ensino Superior de Olinda (Funeso). Recife/PE, Brasil. E-mail: cjackellyne@yahoo.com.br

Antonio Azoubel Antunes - Pós-Doutorado pela Universidade de Pernambuco (UPE); Doutorado em Odontologia pela Universidade de São Paulo (USP). Professor do Corpo Permanente do Mestrado em Perícias Forenses da UPE. Recife/PE, Brasil. E-mail: antonio.antunes@upe.br 